\title{
LONG TERM X-RAY VARIABILITY OF NGC 4151
}

\author{
I. E. PAPADAKIS and I. M. $\mathrm{M}^{C} \mathrm{HARDY}$ \\ Physics Department, Southampton University, \\ University Road, Southampton, SO2 5NH, UK
}

Introduction. Short time scale X-ray power spectra of AGN are in general well fitted by a power law with slopes between -1 and -2 but we expect these slopes to flatten at low frequencies (indication of such a flattening has already been seen in NGC 5506 [1]). We have searched for such a low-frequency break in the power spectrum of NGC 4151 by investigating its long term X-ray light curve (2-10 keV). To construct this light curve we used Ariel V SSI, OSO-8, HEAO-1, Ariel VI, EXOSAT ME and GINGA LAC data.

Method. We have developed a new method to estimate the power spectrum (ps) of this unevenly sampled light curve. This method is based on the "unbiased discrete correlation" technique [2] which we use to estimate the auto-covariance function of the light curve, $D A C F(k)$, at lag $k$. The power spectrum is then estimated by computing the Fourier transform of $D A C F(k)$. In this way the estimated ps is not affected by the "window function" of the uneven sampling pattern.

We have calculated the ps for 8 different bin sizes $(\Delta k=3,6,10,20,30,40,60$, and 80 days). Small bin sizes help us estimate the ps at high frequencies but we have to use larger bin sizes to estimate the ps at low frequencies. We smoothed the 8 individual ps using a simple rectangular window and finally built up the overall ps using contributions from all of them.

Results. We found that our longterm X-ray power spectrum of NGC 4151 is consistent with a power law model with slope of $\sim-2.4$ which flattens at frequencies below $3 \times 10^{-7} \mathrm{~Hz}$. This frequency corresponds to a time scale of $\sim 38$ days.

It is interesting to compare the break frequencies (bf) in Cyg X-1 and NGC 4151. We found that $\mathrm{bf}_{4151} / \mathrm{bf}_{C_{y g} X_{-1}} \sim 7 \times 10^{-6}-8 \times 10^{-7}$ (for Cyg X-1, bf $\sim 0.04-0.37 \mathrm{~Hz}[3])$. If bf in these objects scales proportionally with the central black hole mass, then the black hole mass in NGC 4151 should be between $1.4 \times 10^{6}$ $\mathrm{M}_{\odot}$ and $1.2 \times 10^{7} \mathrm{M}_{\odot}$ (assuming a $10 \mathrm{M}_{\odot}$ black hole in Cyg X-1). If the break frequency corresponds to the viscous time scale of the disk at $\sim 5$ Schwarzchild radii (where most of the X-ray luminosity comes from), then for a $10^{7} \mathrm{M}_{\odot}$ black hole the viscosity parameter should be larger than 0.01 .

References.

[1] McHardy, I.M. 1989, in "Two Topics in X-ray Astronomy", Proc. 23rd ESLAB Symp., Italy, p. 1111

[2] Edelson, R.A. and Krolik, J.H. 1988, ApJ, 333, 646

[3] Belloni, T. and Hasinger, G. 1990, A\&A, 227, L33 